\title{
Herbal Reference Standards
}

Authors

Affiliation
Michael Schwarz, Bernhard Klier, Hartwig Sievers

PhytoLab GmbH \& Co. KG, Vestenbergsgreuth, Germany
Key words

- reference substances

- reference extracts

- phytochemical markers

- herbal medicinal products

- dietary supplements

- food supplements

- quality control

\begin{abstract}
$\nabla$

This review describes the current definitions and regulatory requirements that apply to reference standards that are used to analyse herbal products. It also describes and discusses the current use of reference substances and reference extracts in the European and United States pharmacopoeias.
\end{abstract}

\section{Abbreviations \\ $\nabla$ \\ EP: $\quad$ European Pharmacopoeia \\ HMP: herbal medicinal product}

USP: $\quad$ United States Pharmacopoeia

USP RS: United States Pharmacopoeia reference standard

The term "herbal products" as used throughout this review refers to herbal drugs, herbal preparations as well as to finished herbal medicinal products unless a different meaning is obvious from the context. More specific terms are used where necessary.

Supporting information available online at http://www.thieme-connect.de/ejournals/toc/ plantamedica received Nov. 27, 2008

revised April 9, 2009

accepted April 14, 2009

Bibliography

Dol $10.1055 / \mathrm{s}-0029-1185705$

Published online May 18, 2009

Planta Med 2009; 75: 689-703

(c) Georg Thieme Verlag KG

Stuttgart $\cdot$ New York .

ISSN 0032-0943

\section{Correspondence}

Dr. Michael Schwarz

PhytoLab GmbH \& Co. KG

Dutendorfer Str. 5-7

91487 Vestenbergsgreuth

Germany

Phone: + 49916388327

Fax: +49916388456

michael.schwarz@

phytolab.de

www.phytolab.com

\section{Introduction \\ $\nabla$}

Reference standards are used to calibrate and validate the testing methods that are applied within the framework of quality control throughout all of the stages in the production and manufacture of herbal products. The quality of these reference standards is therefore of prime importance to the quality and associated safety and efficacy of these products. As the individual constituents themselves are only rarely identified in herbal products, the analysts frequently have to rely on characteristic constituents, so-called phytochemical markers. This review therefore begins by outlining the regulatory requirements that apply to the selection and use of markers. It then focuses on the regulatory stipulations and practical requirements that apply to the establishment, characterisation, documentation and handling of the reference standards produced on the basis of these. Reference standards used to test herbal preparations and products are basically subject to the same requirements as those used to analyse chemically defined products. A few special requirements are imposed with respect to the refer- ence standards for herbal products and these are discussed in greater detail below [1-4].

Herbal medicinal products (HMP) have to meet many statutory requirements relating to their manufacture, constitution, testing, storage and distribution. Most of the pertinent specifications and regulations describe the level of requirements and the criteria to be met in order to comply with them. This means, for example, that there are detailed regulations stipulating that the active substance in an HMP must be declared and tested, as well as the way in which this must be done. At the same time, comprehensive validation should ensure that the applied methods provide precise, reproducible and accurate results. The results of analytical methods are affected by numerous influential variables, such as the matrix, the reagents and materials used, as well as light, temperature and equipment, where applicable, and last but not least - the mode of operation. In this respect, there are few factors that are of such fundamental importance and require such exhaustive testing and qualification as the reference standard used. Unlike physical properties, chemical reference standards cannot be traced back to 
international prototypes. Their suitability as points of reference for the determination of a characteristic, e.g., "substance XY content" ensues from a multitude of factors, which can frequently only be determined by means of relative methods. This problem is exacerbated by the diversity of possible sources for one and the same herbal reference standard. The requirement relating to the active substance content of an HMP (accuracy of $\pm 5 \%$ ) is a hurdle that can frequently only be met with a great deal of effort and expense. Traditional herbal combination products typically containing 3 or more herbal preparations are even more challenging. It is not unusual that the marker substances in such combination products are present at a trace level only. Considering the $\pm 50 \%$ tolerances that are usually accepted in trace analysis by way of contrast, the magnitude of the challenge facing guideline-compliant analysis of herbal products today becomes evident.

Alarmingly, little attention is given to the quality of the standards used in pharmacological or toxicological research, on the other hand, in spite of the fact that the test results obtained here often constitute the basis to assess the safety and efficacy of herbal products.

\section{Regulatory Requirements and Definitions}

While the legal framework and detailed quality requirements are clearly regulated for HMP in the EU, in the USA and in many other countries, these have not yet been defined to the same extent for food supplements (covered by food law within the EU) or dietary supplements in the USA (covered by the Dietary Supplements and Health Education Act [5]), and there are good reasons for this. However, as health-promoting functions and effects are being claimed to an increasing extent, it is to be expected that quality requirements for such products be tightened up in the future. This has already taken place in the USA with the introduction of cGMP for dietary supplements in June 2007 [6]. The quality of reference standards and the pertinent documentation play an important role in this respect. In contrast to this, there are no explicit rulings for the quality of plant-based food supplements in the EU, apart from the general quality requirements that apply to all foodstuffs. Nevertheless, the quality aspect has been brought up more frequently in discussion papers and drafts in recent years; examples include the current EFSA draft guidance document for the safety assessment of botanicals and botanical preparations used in food supplements [7] and the European Commission's Terms of Reference for the assessment of claims by the EFSA [8].

As far as the manufacturers of drugs and medicinal products are concerned, the guidelines drawn up by the WHO, ISO and NIST serve as guiding principles, and the monographs of such internationally acknowledged pharmacopoeias as the Pharmacopoeia Europaea (Ph. Eur. or EP) [9] and the United States Pharmacopoeia (USP) [10] are binding within their respective geographical scopes. There are no specific EMEA or ICH guidelines for reference standards yet. A few fundamental requirements for the establishment, documentation and use of primary and secondary standards within the framework of manufacturing and testing active pharmaceutical ingredients (APIs) can be found in the Note for Guidance on Good Manufacturing Practice for Active Pharmaceutical Ingredients (CPMP/ICH/4106/00) [11].

The ISO Guides 30-35 of the International Organisation for Standadization are of essential importance to every manufacturer or user of reference standards [12-17]. Although these documents are not legally binding, they represent the generally accepted international state of the art and therefore constitute the basis for the use of reference standards where no special, mandatory regulations exist. This is the case in the European Pharmacopoeia and USP, for example, in which the chapters on reference standards primarily describe details and conventions relating to the handling of pharmacopoeial standards, while agreeing with the requirements of the ISO Guides to a great extent regarding the technical principles.

- ISO Guide 30: 1992: Terms and definitions used in connection with reference materials.

- ISO Guide 31: 2000: Reference materials - contents of certificates and labels.

- ISO Guide 32: 1997: Calibration in analytical chemistry and use of certified reference materials.

- ISO Guide 33: 2000: Uses of certified reference materials.

- ISO Guide 34: 2000: General requirements for the competence of reference materials producers.

- ISO Guide 35: 2006: Reference materials - general and statistical principles for certification.

ISO Guide 30: 1992 defines a reference material as follows: "Material or substance one or more of whose property values are sufficiently homogeneous and well established to be used for the calibration of an apparatus, the assessment of a measurement method, or for assigning values to materials." At the same time, a reference material can also be a defined individual substance.

ISO Guide 30 defines a certified reference material (CRM) as follows: "Reference material, accompanied by a certificate, one or more of whose property values are certified by a procedure which establishes its traceability to an accurate realization of the unit in which the property values are expressed, and for which each certified value is accompanied by an uncertainty at a stated level of confidence."

Of formal significance is the fact that each set of rules and standards - ISO Guides and EP - contains cross-references to the other one, which means that the requirements of the ISO Guides are especially relevant to the pharmaceutical sector, at least indirectly. This is particularly important where standards that have been established by the manufacturer himself, or a third party, are used for testing instead of pharmacopoeial standards. On the other hand, there are also some important deviations, as neither the EP nor the USP include information about the uncertainty of the assigned value of a reference standard with the respective documentation, "since the precision of the method and uncertainty of the value attributed to the reference standard are taken into account when setting the limit(s) in a monograph" [9].

The stipulations laid down in the EP Section 5.12 "Reference standards" apply to reference standards throughout the area of application of the European Pharmacopoeia, i.e., in the member states of the European Pharmacopoeia Commission (36 states at the moment). Although the rider "This chapter is published for information" is attached to this section, it has a virtually binding effect for the pharmaceutical sector within the pharmacopoeia's scope of application. Any deviations from these stipulations must be documented with evidence of their equivalence.

According to the definition laid down in the European Pharmacopoeia: "Reference standards are shown to be suitable for their intended purpose; they are not necessarily suitable for other purposes. If a reference standard is to be used for any purpose other than that of which it has been established, its suitability for the new use has to be fully demonstrated. Any value assigned to a ref- 
erence standard is valid for the intended use and not necessarily for other uses."

This applies in full consequence to CRS standards of the European Pharmacopoeia. CRS (chemical reference substances) are only regarded as having validity with respect to the tests for which they are specified in the pertinent monographs. The CRS standard specified in an herbal drug monograph must therefore qualify before it can be used to test an extract made from the drug, for example.

Within the European Pharmacopoeia's scope of application, the term "reference standard" is used to refer to reference substances, reference preparations and reference spectra. This means that the reference extracts increasingly used in the testing of herbal products and described in certain pharmacopoeia monographs are equivalent to reference substances by definition.

Reference standards that have been established under the aegis of and released by the European Pharmacopoeia Commission are generally referred to as European Pharmacopoeia Reference Standards. European Pharmacopoeia Chemical Reference Substances are primary reference standards by definition (with the exception of certain antibiotics, which are calibrated in international units).

A primary standard is defined as being: "A standard shown to have suitable properties for the intended use, the demonstration of suitability being made without comparison to an existing standard." Secondary standards are derived by comparison with primary standards. The term working standard is used to describe secondary standards that serve as standards within the framework of routine analysis. They are derived from primary reference standards and are therefore equivalent to secondary reference standards.

The terms internal and external standard do not refer to a qualitative categorisation of a reference standard, but rather to the way in which it is used.

Reference standards offered by the EDQM are established and qualified within the framework of an elaborate process. This regularly includes testing in several laboratories, as well as the performance of inter-laboratory tests. As far as the testing of herbal drugs or preparations is concerned, reference extracts may also be used as CRSs. It is necessary to resort to comprehensively qualified reference substances for the marker to be quantified in order to qualify such reference extracts. Interestingly enough, the pertinent passage of text merely refers to these as "... well characterised samples of active constituents or markers". This formulation fails to stipulate whether these samples refer to primary standards, which should be the case (the problems associated with reference extracts are discussed below).

The EDQM issues a catalogue containing the current batches of its reference standards at regular intervals. A European Pharmacopoeia Reference Standard can be used as long as the batch is listed as a current batch in the catalogue, whereby it may only be used under the conditions (use immediately after opening) and for the purpose specified in the pharmacopoeia. Theoretically, stability testing of a monographed herbal drug or extract would fall within this scope. However, this is not feasible in practice as it is not possible to plan how long the current batch will maintain its "in use" status. As an alternative, a working standard could be derived in sufficient quantities from a CRS, based on a different source of the standard.

United States Pharmacopoeia (USP) rulings on reference standards: The USP runs a comprehensive establishment and qualification programme for the reference standards used in the appli- cation of pharmacopoeial methods. The USP's rulings on reference standards are described in detail in Section $<11>$ of the General Chapters. They differ from the rulings of the EP with respect to certain formal aspects of the establishment and qualification procedures. Interestingly enough, the USP does not distinguish between primary and secondary standards; neither of these terms are used. The categories of standards used by the USP are USP Reference Standards (USP RS) and so-called Authentic Substances (USP AS).

USP Reference Standards are standards that have been established in accordance with an extensive protocol and released for use within the framework of monographs or general methods by the USP Reference Substances Expert Committee. The USP already attaches great importance to the qualification of the source material for a reference standard batch. The currently released reference standard batches are listed on the USP web site (www. usp.org) with the pertinent batch numbers, whereby a distinction is made between current lots and previous lots. A current lot may be used for as long as it remains listed on the web site. If a batch falls into the previous lot category, it is given a "valid use date". The user assumes responsibility for ensuring that he always uses a current lot or a previous lot which is still within the "valid use date". Like the EP CRSs, the user does not receive a certificate of analysis although the label provides comprehensive information concerning the quality.

\section{Selection of Markers for Herbal Medicinal Products $\nabla$}

Unlike chemically defined products, the constituents responsible for the therapeutic activity and efficacy of most herbal products are only known to a certain extent, if at all. This is the reason why the active substance in an HMP (or food/dietary supplement) is always the herbal preparation in its entirety and complexity, e.g., an extract or a plant powder. The use of suitable reference standards for herbal products is therefore preceded by the selection of constituents that are suitable for control purposes, socalled markers. Regulatory stipulations for the selection of markers for herbal medicinal products can be found in various guidance documents issued by the EMEA Herbal Medicinal Product Committee (HMPC) and the FDA. These essentially consist of the following documents:

- Guideline on quality of herbal medicinal products/traditional herbal medicinal products (CPMP/QWP/2819/00 Rev 1) [18].

- Guideline on specifications: test procedures and acceptance criteria for herbal substances, herbal preparations and herbal medicinal products/traditional herbal medicinal products (CPMP/QWP/2820/00 Rev 1) [19].

These stipulations include the following: "In the case of herbal substances with constituents of known therapeutic activity, assays of their content (with the test procedures) are required ..." and "...where constituents of known therapeutic activity are not known, assays of marker substances (with the test procedures) are required. The choice of markers should be justified."

And for herbal preparations: "A quantitative determination (assay) of markers or of substances with known therapeutic activity is also required. The content should be indicated with the lowest possible tolerance (the narrowest possible tolerance with both upper and lower limits stated). The test methods should be described in detail." 
These requirements apply analogously to finished products. Markers are defined as follows: "Markers are chemically defined constituents of a herbal substance which are of interest for control purposes independent of whether they have any therapeutic activity or not. Markers may serve to calculate the quantity of herbal substance(s) or herbal preparation(s) in the finished product if that marker has been quantitatively determined in the herbal substance(s) or herbal preparation(s) when the starting materials were tested. There are two categories of markers: Active markers are constituents or groups of constituents which are generally accepted to contribute to the therapeutic activity. Analytical markers are constituents or groups of constituents that serve for analytical purposes."

Comparable requirements are stipulated in the FDA's "Guidance for Industry - Botanical Drug Products" [20].

While markers for many herbal drugs and drug preparations are defined in the pertinent monographs of the European Pharmacopoeia (or other acknowledged pharmacopoeias, e.g., USP, BP, PF, etc.), markers for herbal drug preparations that have not been monographed can often only be selected in the course of developing the product or method. As far as extracts are concerned, the categories in accordance with the EP "Extracts" monograph play an important role:

Standardised extracts are adjusted within an acceptable tolerance to a given content of constituents with known therapeutic activity.

Quantified extracts are adjusted to a defined range of constituents.

Other extracts are essentially defined by their production process.

Suitable markers must first be selected and established, particularly where constituents responsible for or contributing to the therapeutic activity are unknown. Important criteria required for this have been proposed in the recent HMPC "Reflection Paper on Markers used for Quantitative and Qualitative Analysis of Herbal Medicinal Products and Traditional Herbal Medicinal Products" (EMEA/HMPC/253 629/2007) [21]:

- Characteristic for the herbal drug;

- Constancy of presence in the herbal drug/preparation;

- Chemically defined;

- Analysable with routine analytical equipment;

- Adequate content in herbal drug and herbal drug preparation for the development of a valid, reproducible quantitative method;

- Stability.

While these criteria define the framework for selection, characterisation and use of marker substances for herbal medicinal products, there are currently no corresponding sets of rules for plant-based foodstuffs, such as functional food and food supplements. However, principles existing for markers of herbal medicinal products could be applied analogously where appropriate.

\section{Techniques for the Selection, Identification and Characterisation of Marker Substances in Herbal Preparations}

A highly developed set of analytical methods and instruments are currently available for the selection and identification of characteristic markers in herbal preparations. Although this is not intended as a subject for detailed discussion in this article, it has vi- tal importance for herbal products in particular, for the reasons mentioned in the introduction. The purely phytochemical screening procedures used to locate characteristic secondary substances by combining TLC, HPLC or GC with various classical detection methods (e.g., chromogenic reagents, UV-VIS, FID, etc.) in the past are now being used alongside more specific methods, such as GC-MS, LC-MS/MS or LC-NMR. These methods offer a very rapid means of characterising or even identifying secondary substances in complex fractions or complete extracts without isolating the substances concerned beforehand. They therefore offer a means of identifying minor components, as well, which could only be determined in very time-consuming processes using larger quantities of plant material until just a few years ago. Constituents that contribute towards therapeutic activity can also be selected and characterised or identified on an analytical scale by means of bioassay-guided fractionation. In this context, the development of informative natural substance spectral libraries is becoming increasingly important. Nowadays, numerous spectral libraries are available for different measuring techniques, whereby LC-MS libraries play a major role in the identification of natural substances. This approach enables a high level of dereplication for the isolation and structural determination of natural substances on a preparative scale. The informational value of an automated comparison with spectral libraries is fundamentally subject to certain restrictions. The signal patterns and intensities of mass spectra depend on analysis conditions and matrix effects in some respects, even if the ionisation technique is the same. This can give rise to false positive or false negative assignments or exclusions, particularly where large, pooled spectral databases are concerned. Nevertheless LC-MS screening is primarily used for selection of suitable markers. As a rule, fully documented structural determination with subsequent establishment of a reference substance is achieved by carrying out off-line measurements on appropriately large quantities of the substance and a comprehensive comparison with published reference values for different measuring techniques (UV, IR, NMR, MS, etc.).

Examples of commercially and/or publicly accessible spectral databases with high-quality data are as follows:

- NIST/EPA/NIH (NIST 08) Mass Spectral Library [22];

- Spectral Database for Organic Compounds SDBS [23].

\section{Requirements for the Characterisation of Primary Standards \\ $\nabla$}

\section{Identification}

The analytical characterisation of primary reference substances is subject to the most stringent requirements. The identity of a primary reference substance is verified through characterisation by appropriate chemical attributes such as structural formula, empirical formula and molecular weight are (also refer to Section 5.12 of the European Pharmacopoeia). Nuclear magnetic resonance (NMR) spectroscopy performs a central role in identity testing as the structure of the reference substance can be determined by measuring and interpreting 1D- and 2D-NMR spectra without the need for comparison with reference spectra. Apart from the usual ${ }^{1} \mathrm{H}$ - and ${ }^{13} \mathrm{C}-\mathrm{NMR}$ spectra, which generally suffice for structural determination, ${ }^{14 / 15} \mathrm{~N}-,{ }^{17} \mathrm{O}$ - and ${ }^{31} \mathrm{P}-\mathrm{NMR}$ spectra may be measured and interpreted for verification or validation of specific structural elements containing heteroatoms. Although $\mathrm{X}$-ray structural analysis also offers a suitable alternative for in- 
dependent determination of the molecular structure, this technique is in less widespread use and requires the substance to be crystallised. Information about the molecular weight of the reference substance can be obtained by recording mass spectra and the measurement of high-resolution spectra may enable conclusions to be drawn regarding the empirical formula. Substancetypical fragmentation patterns emerge according to the ionisation technique used, which enable interpretations relating to the underlying structural units and the presence of certain substituents. Complete determination of the structure is seldom possible by means of mass spectrometry alone, however, which means that the method can only be used to verify the identity of a primary standard in conjunction with other methods. The same applies analogously to UV and IR spectra. Although conclusions may be drawn from both types of spectra regarding certain structuredetermining properties, with IR spectra demonstrating a substance-specific fingerprint region in addition to this, neither of them ever provide sufficient information to deduce and verify the complete structure. These spectra must always be either compared with a spectra library or combined with other identity tests. Apart from the methods mentioned above, the melting point can also be determined, but this may be subject to severe fluctuations depending on the last solvent used and the crystal structure. It may be necessary to determine the optical rotation value and/or record $C D$ spectra for compounds with chiral centres. While identity testing by means of TLC requires a reference standard for comparison, performance of an elemental analysis may also prove helpful in confirming the elemental composition. Deviations from the composition or mass differences as anticipated on the basis of mass and NMR spectra may indicate possible impurities, such as water, residual solvents or inorganic constituents.

\section{Purity testing}

Chromatographic methods: A combination of various analytical procedures is also required to determine the purity of a primary reference substance. Organic impurities in the reference substance are determined by means of a selective separating procedure - usually chromatographic (HPLC or GC) or electrophoretic (CE) - combined with a suitable detection technique. Attention must be given to ensuring that certain impurities are not left unaccounted for by the selected separating technique, e.g., non-volatile impurities in the event of gas chromatography or uncharged molecules where CE is used. UV or diode array detectors are primarily used with HPLC methods as they represent detection technologies that are fairly universal, as well as sensitive. The chromatograms or electropherograms are usually evaluated according to the area normalisation method, i.e., an area percentage evaluation is carried out on all of the signals recorded in a chromatogram or electropherogram while allowing for the blank value. The nature of this method of evaluation is such that it is susceptible to a methodical error as it presupposes that all detected compounds have identical response factors. To eliminate this error, all of the impurities in the substance must first be known or identified and must then be quantified exactly with the help of a specific primary reference standard. The effort and expense involved in completely isolating and identifying potential impurities is unaffordable for natural substances because of the complex compositions of herbal drugs and, if at all, this approach may only be considered for synthetic chemical compounds, with educts and synthesis by-products known and available in sufficient quantities. The nature of the area normalisation (100\%) method is such that the purer the reference substance, the smaller the error. In addition to this, the error is reduced to a minimum if one assumes that mostly impurities, which are closely related in terms of structure and therefore have similar UV spectra and response factors, are co-extracted in the course of isolating the reference substance. UV detection is particularly suitable for application of the area normalisation method because related compounds usually have similar UV response factors that are not dimensions apart from that of the reference substance, which could be the case for electrochemical detection or detection by mass spectrometry. The suitable detection wavelength must be selected after recording the UV spectra of the reference substance and any secondary peaks. Where possible, the wavelength should be an absorption maximum that includes all of the chromatogram or electropherogram signals. Many natural substances (e.g., saponins) lack a pronounced UV chromophore and their UV spectra merely show a steeply declining shoulder around $200 \mathrm{~nm}$. Nevertheless, detection in the low-UV range (using suitable UV-transparent solvents) should be given precedence over mass spectrometric or light-scattering detection for these compounds as well. A prerequisite for detection by mass spectrometry is that the ionisation capability of many compounds varies tremendously and is extremely dependent on the appropriate functional groups and the ionisation technique used. Although light-scattering detection is a universal method, the detector's linearity range is very small compared with that of a UV detector and this method is therefore less suitable for the parallel detection and quantitation of minor and major components. Another universal technique, refractive index detection (RID), is not used to test the purity of reference substances because of its lack of sensitivity. However, overselective detection techniques, which may not detect impurities under certain circumstances - such as electrochemical detection or fluorometric detection - should not be used either. Derivatisation techniques (e.g., used to enable fluorometric detection for HPLC or to increase volatility as a prerequisite for gas-chromatographic analysis) should also be avoided, as impurities that are not accessible to the derivatisation reaction are masked out by this process.

Universal detectors should also be used for gas-chromatographic purity testing. The flame-ionisation detector (FID) is a particularly suitable one. Various response factors falsify the results in a similar way as in UV detection, however, which depend on the elementary composition and molecular structure of the analytes. The coupling of gas chromatography with mass spectrometric detection by means of electron impact ionisation offers a better alternative for presentation of the chromatogram than HPLC-MS. Selective GC detectors, such as ECD, NPD, FPD or AED, should be avoided. In a similar way to RID in HPLC, the thermal conductivity detector (TCD) used in GC is universal, but does not have the sensitivity needed for precise, quantitative purity testing.

Particular attention should always be given to the concentration of the solution used for purity testing. The chosen concentration must be sufficiently high to ensure that impurities in the $<1 \%$ range also provide adequate detector signals for quantitation. A second, complementary separation technique can be used to verify that the selected chromatographic system offers a suitable means of determining the purity of a primary reference substance. Although not stipulated in any pharmacopoeia or international guideline, this is required by some drug registration and regulatory authorities for the primary standard documentation submitted together with the registration dossier. Ideally, these should be two completely different techniques, such as a GC 
method combined with an HPLC method. If two identical chromatographic techniques are used, on the other hand, the methods should differ in terms of the composition of the mobile phase (HPLC: e.g., methanol vs. acetonitrile; GC: a different carrier gas) and the stationary phase (HPLC: e.g., $\mathrm{C} 18$ vs. $\mathrm{C} 8, \mathrm{NH}_{2}$, diol, phenyl-hexyl, etc.; GC: e.g., polysiloxane vs. polyethylene glycol). Furthermore, in the case of two HPLC methods, two different detection wavelengths should be used, where expedient and if the substance has several UV maxima.

Water and residual solvents: Chromatographic or electrophoretic purity testing is always supplemented by a determination of the water and residual solvent content. The water content can be determined by means of Karl Fischer microtitration and the residual solvent content can be determined by means of headspace GC analysis. Both processes consume only relatively small amounts of the substance. Glycosidic compounds, in particular, form hydrates usually containing at least one extra molecule of water per sugar residue, which can quickly lead to significant water content values between 5 and $10 \%$. The authors recommend that such compounds should not be dried too harshly to eliminate all traces of water, as they otherwise adsorb moisture from the atmosphere and form undefined hydrates when the vessel is opened. The water content of saturated compounds, on the other hand, usually remains stable over a longer period. One example of this type of compound is hyperoside (= quercetin 3galactoside).

In rare cases, pharmacopoeias also contain reference substances in hydrate form. One notable example of these is quercetin dihydrate, which is mentioned in the European Pharmacopoeia monographs on Ginkgo leaf and Ginkgo dry extract refined and quantified and is used to calculate the flavone glycoside content. However, the formula used to calculate the content refers to the content of anhydrous quercetin. As quercetin is commercially available in anhydrous form and is decidedly stable, the detour via quercetin dihydrate is incomprehensible from an analytical point of view.

Determination of the residual solvent content is just as important, as many natural substances tend to incorporate the solvents used within the framework of extraction, recrystallisation or synthesis. Therefore knowledge on the solvents used in the course of these processes can be a great advantage. If this is not the case, it may be possible to identify unknown signals in the gas chromatogram by evaluating the NMR spectra of the substance under investigation, if such are available or can be obtained. One excellent example that demonstrates the problems associated with residual solvents resulting from the widespread use of the reference substance is the hypericin found in St. John's wort (Hypericum perforatum). Hypericin incorporates pyridine very well - according to the experience of the authors hypericin in crystalline form is capable of containing up to $50 \%$ pyridine and this cannot be removed by means of drying processes. Other substances known to show similar effects include anisatin from Illicium anisatum (MTBE or diethyl ether) and silybinin from Silybum marianum (isopropyl alcohol). Instead of determining water and residual solvent content separately, the EP also provides for determination of loss on drying as an alternative, but preference should be given to determining the individual values - from the point of view of substance consumption as well.

Inorganic impurities: Most inorganic impurities are salts that were used during the synthesis or extraction process and have not been completely removed, or silicon in the form of silica gel that was used during chromatographic purification stages. Iso- lated natural substances are also frequently present in salt form. This may be due to the fact that a counter-ion is needed for a structure-specific charge at the core molecule, or for stability reasons, or to facilitate handling, if only the salts of a compound occur in crystalline form, or simply by virtue of the extraction or synthesis process. Examples include the ammonium salts or metal salts of glycyrrhizin, the chlorides of diverse alkaloids (e.g., berberine) or anthocyanins/anthocyanidins (e.g., cyanidin 3-glucoside/cyanidin), or the glucosinolates usually isolated as potassium salts. Only in rare cases does the actual content of such inorganic constituents correspond exactly to the stoichiometric ratio calculated according to the empirical formula. This means that exact determination of the inorganic constituents is absolutely essential for characterisation as a primary standard. This also appears expedient from the point of view that, as a rule, the content of a constituent substance in a drug or preparation is the information that is of interest, not the content of a certain salt, which is not usually present in the herbal preparation in this form anyway (many inorganic ions or organic acids may be present as counter-ions in the herbal matrix). Examples of this include berberine chloride or cyanidin chloride, both of which are also available from the EDQM in the form of CRS reference substances. While the monographs making use of berberine chloride include the percentage content of chloride-free substance in the calculation formulae, this is not the case for cyanidin chloride or cyanidin 3-glucoside chloride. Instead, the respective chloride salt content is calculated for both of these, a factor that does not seem expedient for the reasons given above.

ICP-MS is a frequently used and convenient method for the simultaneous determination of multiple elements. Although just as suitable, AAS does not permit the determination of several elements in parallel. Argentometric titration constitutes a classical and practical method of determining halides. Ion chromatography (IC) also enables the simultaneous determination of the most diverse ions. All of these methods are characterised by the fact that they can be carried out on a micro scale and only use small quantities of material. Occasionally required by registration authorities, the method of establishing inorganic impurity content by determining the ash content in accordance with pharmacopoeial specifications, on the other hand, is unsuitable as a rule because this method requires at least $1 \mathrm{~g}$ of material.

Content assignment: All of the methods used to test purity as described above must have been validated to an adequate extent [24]. Evidence must be provided of the selectivity (e.g., measuring a blank in combination with peak purity determination), linearity (at least 5 measuring points, generally within the range between approx. 50 and $150 \%$ of the concentration used for the $100 \%$ value) and repeatability (at least 6 injections) of the chromatographic or electrophoretic method used. The assigned content is then calculated according to the following formula:

Assigned content $=[100 \%-($ water + residual solvent + inorganic impurities)] $\times$ chromatographic/electrophoretic purity (\%)

If two complementary techniques are used, the calculation is made on the basis of the more selective method or the mean value for both methods, given that no significant discrepancies between the results of both methods have been found by means of suitable statistical tests.

Appropriate absolute methods may also be used for purity testing and content assignment, although virtually none of these are suitable for universal application and all of them have disadvan- 
tages. Examples of these include DSC (differential scanning calorimetry, which cannot be used for substances that decompose on melting) and phase solubility analysis (time-consuming, decomposition processes, polymorphism of the main component). Other methods, which are generally only group-specific and therefore unsuitable for content determination without simultaneously testing for related impurities, include UV spectroscopy (primary reference substance or knowledge of the extinction coefficient determined by means of a primary reference substance required, nonselective), optical activity (primary reference substance or knowledge of the specific angle of rotation determined by means of a primary reference substance required, nonselective, poor sensitivity) or titrations. Generally speaking, methods like these are merely used to confirm the results of the more specific chromatographic/electrophoretic purity tests and are not included in the assignment of the content.

Another method for determining the absolute content is quantitative nuclear magnetic resonance spectroscopy (qNMR). Even this technique requires sophisticated and expensive instrumentation currently limiting its broader use, it offers many unique and desirable features. They are described in several excellent reviews in detail already [25-27], and one contribution to this issue will elaborate on an application of qNMR. Nevertheless, a few cornerstones of this technique should be briefly mentioned here as well. Besides being nondestructive, fast, and selective, qNMR is the only approach providing simultaneous access to the chemical structure as well as quantitative information. The NMR signal is directly proportional to the number of nuclei in the structure; thus, the true composition of the sample will be reflected, regardless the diverse physicochemical properties of its constituents (e.g., UV absorption). This is especially helpful for an exact characterisation of so-called "pure" reference compounds, but also applicable to multicomponent matrices such as food items and natural products $[28,29]$. A fine example for the first problem is a recently reported study by Mohn et al. [30], which compares the purity assessment of isolated glucosinolates from Isatis tinctoria by qHNMR and HPLC. Analysis of the compounds by the latter indicated purities of $99 \%$ and above, whereas qHNMR data revealed much lower values of 60 to $90 \%$; one compound (4-hydroxyglucobrassicin) showed a purity of $17 \%$ only.

Needless to say that qNMR has certain limitations as well. They are, for example, a sometimes insufficient sensitivity (constituents can usually be determined down to a content of $1 \%$ with $1 \%$ error; [27]), the need for calibrants and the fact that experienced operators are required in order to get optimum results. However, recent developments like automated sample preparation for high-throughput analysis, advanced referencing techniques like ERETIC and ARTSI [31], and an ever increasing number of successful applications already have converted this method from a purely scientific one to a well reputed and established approach. This transition might also be indicated by the fact that qNMR will be included as assay procedure in the next revision of the European Pharmacopoeia [4].

\section{Requirements for the Characterisation of a Secondary Chemical Reference Standard}

$\nabla$

Secondary standards, which are frequently also referred to as working standards, are always derived from primary reference standards and must demonstrate identical characteristics to the primary standards with respect to the tests for which they are used. Apart from this, traceability must also be assured. The necessary scope of testing primarily depends on the intended purpose. If the secondary standard is to be used for IR identification, for example, then its IR spectrum must be identical to the IR spectrum of the primary standard. If the secondary standard is to be used for identification purposes within the framework of chromatographic or electrophoretic methods, the European Pharmacopoeia requires conformity with respect to the migration distance (TLC, electrophoresis), migration time (CE) or retention time (HPLC, GC). Considering the fact that not only highly pure substances are used as secondary standards, but frequently also extracts or less pure substances, which may contain impurities with similar characteristics, these requirements are not always sufficient for practical application. The identity of a signal should at least be confirmed by another selective method, whereby a mass spectrometric analysis offers an expedient solution as a rule. Primary and secondary standard intercalibrations must be carried out in order to determine the content of a secondary standard. The European Pharmacopoeia does not lay down any explicit requirements in this respect; it merely stipulates that the number of independent repetitions and the acceptance criteria are defined in the preliminary stages. An approach that has proved expedient in practice involves preparing two parent solutions of both standards and measuring each solution at least 6 times. The acceptance criteria must have been specified in advance, whereby the variations resulting from the apparatus must be taken into consideration, e.g., the repeatability of an autosampler. A common acceptance criterion is a variation coefficient of $<2 \%$ referred to the peak areas corrected with respect to concentration; this also appears in USP monographs as an acceptance criterion for the relative standard deviation obtained from repeated injections of the same sample.

\section{Primary and Secondary Standards in Practice $\nabla$}

In practice, the question arises as to whether the system of primary and secondary standards is practical in the given situation and whether the associated effort and expense is reasonable. A comprehensively characterised high purity primary standard is always needed and a secondary standard must be calibrated at regular intervals in addition to this. Evidence of the primary standard's stability must also be provided in the form of a retesting programme. This includes regular reexamination of the chromatographic purity, supplemented by repeated testing of the water and residual solvent contents, where applicable. Additional costs are incurred by the analytical work involved and the associated consumption of substances. Apart from this, adequate quantities of the primary standard must be available right from the beginning to avoid a situation in which a new primary standard has to be established at regular intervals. It is important to be conscious of the fact that the content value determined for the secondary standard constitutes a black box to a certain extent: no details of the individual analytical values (water content, residual solvent content, etc.) are available. If problems are encountered in the course of analysis, there is no way of selectively determining the cause and of verifying and remedying the problem by means of new analytical data (e.g., increased water content due to the hygroscopicity of the reference standard used). Instead, the secondary standard has to be recalibrated against the primary standard. In this case, problems may be encountered 
with respect to the availability of the batch of primary standard that was originally used for calibration.

It may prove more reasonable to use just one reference substance and to characterise this to an adequate extent. There is certainly no need to use a combination of all methods that are available (see above) to verify the identity of a reference substance used in routine analysis. Comprehensive identification can take place when the (primary) standard is established for the first time (e.g., with a combination of NMR, MS and IR), while for consecutive batches a comparison of the IR spectra is sufficient to verify the identity. As far as quantitation is concerned, the determination of chromatographic purity, as well as the water, residual solvent and inorganic impurity content are obligatory. The user then has a sufficiently characterised substance at his disposal, for which quality can be assured by means of a retesting programme. This approach avoids the need for maintaining batches of both primary and secondary standards and the associated effort and expense. Such a substance can also be classified as being a primary reference substance in accordance with the definition laid down in the European Pharmacopoeia as all of the data required for content assignment is available and a "relevant" part of possible identity verification tests as outlined in Section 5.12 of the EP has been carried out.

There is no uniform use of the terms "primary standard" and "secondary standard" in practice and they are unfortunately used misleadingly on some occasions. The term "secondary standard", in particular, is frequently used when referring to a substance which has only undergone chromatographic purity testing. Although such substances may have been characterised to a completely adequate extent for many applications (e.g., for initial screening procedures, preliminary tests, etc.), they do not conform to the official definitions of secondary standards.

\section{Reference Extracts}

$\nabla$

The use of herbal reference extracts instead of pure reference substances is a very controversial issue. Some reference extracts have recently been included in the European and United States Pharmacopoeias. One clear argument in favour of extracts is the easy availability of larger quantities and the relatively low price, as compared to the price of an isolated pure substance. The use of reference extracts does have its disadvantages, however. This means, for example, that the assigned values, which depend on the concentration of the analyte and the chromatographic resolution, are subject to greater statistical uncertainty than is the case when a pure substance is used [32]. Many extracts are hygroscopic; a property that imposes high requirements on the packaging, handling and storage processes. As far as analysis is concerned, the solubility of extracts can present a problem as a result of matrix components. Retention time shifts and peak broadening caused by matrix effects cannot be ruled out either. Reference extracts have not necessarily been produced according to the same method as the extract under examination, which means that matrix differences can occur, possibly resulting in variations of the chromatographic resolution. It is therefore absolutely essential that the chromatographic method used to determine the content value of the reference extract is identical to the one subsequently used to control the quality of the product. This requirement imposes major restrictions on the possible applications of a reference extract in other test procedures. The definition of longterm stability values for reference extracts, which must be based on stability tests, of course, also presents difficulties. Storage at $-80^{\circ} \mathrm{C}$, a temperature at which one can generally assume that chemical compounds are stable, is not feasible for many users with a reasonable amount of effort and expense. This means, for example, that a primary standard is needed to provide the necessary evidence of stability, as well as to establish the marker content in the reference extract.

The use of a reference extracts therefore only appears expedient if several of the following prerequisites are met:

- the isolation or procurement of the pure substance involves an unreasonable amount of effort and expense;

- in pure form, the pure substance is not sufficiently stable;

- the extract used permits a robust chromatographic baseline separation;

- the analyte exhibits a high concentration in the extract;

- the extract has a high physical and chemical stability;

- an analysis requires several reference substances, which are already jointly present in the extract.

It is generally true to say that a reference extract is always a derived or secondary standard, which requires a primary reference substance for initial establishment. This means that no other (additional) secondary standard can be derived from a reference extract.

\section{CRS reference extracts: characterisation requirements} and practical consequences

EP chemical reference standards are regarded as being primary standards by definition and, as a rule, this is acknowledged by regulatory authorities. The classification of CRS reference extracts as primary standards is a contradiction in terms, however, as they do not fulfil the criteria for a primary reference standard according to the pertinent international definitions (or those of the EP). When a CRS reference extract is established for quantitative purposes, the content is determined by calibrating the extract against a pure substance, which means that the reference extract is actually a derived standard. The extract is then designated as a "primary standard" by definition. As for any secondary standard, the establishment of CRS reference extracts should therefore be subject to the requirement that a primary standard must always be used for content quantitation. As mentioned in the introduction, the European Pharmacopoeia merely specifies that "...well characterised samples of active constituents or markers" must be used to qualify CRS reference extracts. This appears to be inadequate, as a requirement stipulating the use of a primary standard would be needed. No detailed procedure for the qualification of CRS reference extracts has been described to date. Content assignment takes the form of a "collaborative trial", but Section 5.12 of the EP does not describe the nature of this or mention the number of parties involved in any greater detail. If no primary standard is used for content quantitation of CRS reference extracts, there is a risk that deviating content values could be found in a new batch of a CRS reference extract, which may possibly have been produced with a new batch of the pure substance, whereby these deviations may be caused by fluctuations in the impurity profile of the standard used for calibration. This could then result in deviating content values for the products tested by means of the CRS reference extracts, which could lead to complications, e.g., with respect to compliance with specifications. Special attention should also be given to the chromatographic resolution of relevant peaks of the CRS reference extracts. The pertinent extract monographs often fail to exactly define the 
Table 1 CRS reference extracts in the European Pharmacopoeia (Issue 6.3).

\begin{tabular}{|c|c|c|c|c|c|c|c|}
\hline \multirow{2}{*}{$\begin{array}{l}\text { CRS extract } \\
\text { in EP }\end{array}$} & \multicolumn{6}{|c|}{ Criteria for establishing a reference extracts } & \multirow{2}{*}{$\begin{array}{l}\text { Alternative to } \\
\text { the extract in } \\
\text { the USP }\end{array}$} \\
\hline & $\begin{array}{l}\text { Pure substance } \\
\text { unavailable or } \\
\text { expensive }\end{array}$ & $\begin{array}{l}\text { Pure sub- } \\
\text { stance } \\
\text { unstable }\end{array}$ & $\begin{array}{l}\text { Extract permits } \\
\text { robust chrom. } \\
\text { separation }\end{array}$ & $\begin{array}{l}\text { High concentra- } \\
\text { tion of the analyte } \\
\text { in the CRS extract }\end{array}$ & $\begin{array}{l}\text { Extract } \\
\text { stable }\end{array}$ & $\begin{array}{l}\text { Analysis requires } \\
\text { several refer- } \\
\text { ence substances }\end{array}$ & \\
\hline Senna & - & - & not required & not required & $\begin{array}{l}\text { limited } \\
\text { importance }\end{array}$ & - & Sennosides \\
\hline Ginkgo & $\mathrm{O}^{1}$ & - & $\begin{array}{l}\text { limited } \\
\text { importance }\end{array}$ & $\begin{array}{l}\text { limited } \\
\text { importance }\end{array}$ & $\begin{array}{l}\text { limited } \\
\text { importance }\end{array}$ & + & $\begin{array}{l}\text { Ginkgo Terpene } \\
\text { Lactones }\end{array}$ \\
\hline Valerian & - & - & + & $-(0.45 \%)$ & $?$ & - & Valerenic acid \\
\hline Milk Thistle & - & - & - & + & $?$ & + & Silybinin \\
\hline Ivy Tincture & - & - & + & $-(0.20 \%)$ & $?$ & - & No monograph \\
\hline Chaste Tree & - & - & - & $-(0.13 \%)$ & $?$ & - & Casticin \\
\hline St. John's Wort & $0^{1}$ & $\mathrm{O}^{2}$ & + & $-(0.050 \%)$ & $?$ & + & Oxybenzone \\
\hline Bilberry & $\mathrm{O}^{1}$ & $\mathrm{O}^{2}$ & $\mathrm{O}^{3}$ & $-(3.44 \%)$ & $?$ & + & $\begin{array}{l}\text { Cyanidin 3-glu- } \\
\text { coside chloride }\end{array}$ \\
\hline Artichoke & - & - & + & not required & $\begin{array}{l}\text { limited } \\
\text { importance }\end{array}$ & - & - \\
\hline
\end{tabular}

${ }^{1}$ All substances are commercially available but are priced comparably higher than others. ${ }^{2}$ Stable if stored at $-20^{\circ} \mathrm{C} .{ }^{3} \mathrm{Cyanidin} 3$-glucoside chloride shows a well isolated peak, but other signals are not ideally separated

extraction solvent used to produce the extract. Extract variants that are within the framework defined by the monograph can therefore bring about a situation in which critical peak pairs are present in relationships that deviate from the CRS reference extract. Any problems associated with the resolution of critical peak pairs, which could result from this, should already be avoided when CRS reference extracts are established. This could be achieved by analysing commercial extract samples that represent the variability of the extraction solvent within the framework of the monograph, for example. In many cases, the composition of the herbal drug used to produce the extract, which is subject to natural fluctuations, can exert just as much variability as the extraction solvent. Evidence of the stability of CRS extracts must be provided by calibrating them against primary reference substances at regular intervals.

All CRS reference standards are intended and suited for single use only. This particularly applies to the use of CRS extracts, as the user, without a primary reference substance of his own, has virtually no means of monitoring the stability of the used reference extract once the container has been opened. As a consequence reference extracts have very limited suitability to serve as a standard within the framework of long-term stability testing schemes. Formally it is not allowable to establish a secondary reference extract against a CRS extract according to international definitions and this is only currently possible by virtue of the fact that CRS extracts are regarded as being primary standards within the pharmacopoeia convention. Due to the measuring uncertainty associated with repeated calibration between secondary standards users should refrain from establishing a "tertiary" reference extract. Unlike a pure substance, which may be used for testing raw materials, extracts and finished medicinal products, the use of reference extracts is subject to certain restrictions. Generally speaking, the use of officinal standards is limited to the relevant monograph and the method defined there. A monographed method - and the reference extract concerned - may only be used to analyse a finished HMP if the user has validated the method for this additional purpose. A method often has to be modified for combination preparations, in particular, because of their complex composition, and this prohibits the use of a CRS extract unless the user can prove that the CRS extract also exhibits the same content according to the modified method. Apart from this, attention must be given to the fact that, because of the low analyte content in the current CRS extracts, the determined content value is usually subject to higher standard deviations than that of a pure substance, which makes it difficult to comply with the specified stability criteria for the finished medicinal product.

\section{Overview of the current use of reference extracts,} reference substances and reagents in the EP and USP The current edition of the European Pharmacopoeia lists eight CRS reference extracts and one CRS reference tincture ( $\bullet$ Table 1): the senna extract is only used as a reference for identification by means of thin-layer chromatography at the moment, a photometric method is used for the assay. The Ginkgo extract is exclusively dedicated for peak assignment of the terpene lactones ginkgolides A, B, C and bilobalide. Quantitation takes place against benzyl alcohol CRS using response factors. Chlorogenic acid $\mathrm{R}$ and rutin $\mathrm{R}$ are used for identification in the monograph, on the other hand, whereas the quercetin dihydrate CRS already mentioned above is used for quantitation of the flavonoids. A "ginkgolic acid CRS" is used for a ginkgolic acid limit test. This is also a reference extract containing $0.35 \%$ ginkgolic acids.

The "valerian standardised dry extract CRS" serves to calculate the sesquiterpene acid content (sum of valerenic acid and acetoxyvalerenic acid), which is calculated as valerenic acid, whereas reagent grade valerenic acid and acetoxyvalerenic acid are used for identity testing. The "milk thistle standardised dry extract CRS" serves to calculate the total silymarin content (sum of silybinin A and B, isosilybinin A and B, silychristin and silydianin), as well as the percentage content of silybinin, isosilybinin and the sum of silychristin and silydianin, whereas reagent quality silybinin (and taxifolin) are used for identification by means of thinlayer chromatography.

Two reagents - hederacoside $C$ and alpha-hederin - are also used for identity testing in the "ivy leaf" monograph, while the heder- 
acoside $\mathrm{C}$ content is determined with the help of the "ivy leaf standardised tincture CRS".

Two reagents - aucubin and agnuside - are used to test the identity of chaste tree fruits, while the casticin content is determined with the help of the "agnus castus fruit standardised dry extract". Just recently the EDQM established a St. John's wort CRS extract for the determination of total hypericin (sum of hypericin and pseudohypericin), a bilberry extract for determination of total anthocyanins, calculated as cyanidin 3-glucoside chloride, and an artichoke extract for use in a system suitability test (requirement for the resolution between chlorogenic acid and the subsequent peak in the monograph on artichoke leaf dry extract) were established. The St. John's wort extract CRS contains only a very low level of hypericin (0.050\%). Because of the solvent problem already described above special attention must be given to the characterisation of the underlying primary standard used during the establishment of this CRS extract. The authors were able to detect pronounced fluctuations in the quality of hypericin reference substances available on the market within the framework of their own investigations. This was only ever possible by means of a direct peak area comparison of the prepared solutions as all of the tested samples exhibited high HPLC purity levels in excess of $97 \%$. However, a comparison of the concentration-corrected peak areas with a primary reference substance showed that the hypericin samples exhibit an absolute content of just 3\% in extreme cases. Apart from the high residual solvent content, another possible explanation for this phenomenon is the presence of polymeric hypericins, which cannot be detected by means of HPLC analysis as they would merely give rise to a generally higher baseline in the chromatogram rather than producing any discrete peaks. Likewise, attention should be paid to the stability of the bilberry reference extract, because anthocyanins have a pronounced tendency towards polymerisation reactions.

Since 2004, the European Directorate for the Quality of Medicines (EDQM) has made considerable efforts in order to establish herbal CRS of which only a very limited number were available in the past. Today already 24 CRS substances are used for the analysis of herbal drugs and extracts, most of which for assays (- Table 2 ). The European Pharmacopoeia contains a considerably larger number of reagents that are not only used for identity testing, but also for assays in 26 cases ( Table 3). Action is clearly needed here in order to establish CRS reference substances. As a rule, the requirements that currently apply to reagents are limited to chromatographic testing by means of GC or HPLC, which must result in between 90 and 99\% purity. The calculation formula for the pertinent assay usually allows for the chromatographic purity of the standard with the exception of the few photometric assays. The number of reagents used for identity testing without these "fine chemicals" having to meet any requirements for identity is much higher. It is noticeable that, in some cases, reagent quality substances are used for assays in spite of the fact that corresponding CRS substances would be available (e.g., ferulic acid R in Eleutherococcus but ferulic acid CRS in Lemon verbena leaf, quercetin dihydrate R in Ginkgo leaf but quercetin dihydrate CRS in "ginkgo dry extract refined and quantified"). Hederacoside $\mathrm{R}$ is used for the assay in the monograph "Hedera helix for homeopathic preparations", whereas a CRS tincture is used for ivy leaf, as described above.

Interestingly enough, the USP gives the use of reference substances precedence over the use of reference extracts unless the pertinent reference substance is not commercially available, or it is unstable or unusually expensive. In these cases, a reference ex- tract is used that is calibrated against primary standards and is subsequently subject to the USP RS Continued Suitability of Use Program. Sixteen reference extracts are currently in use in the USP ( Table 4). However, only four of these in total (two exclusively) are used for assays. Fourteen of the 16 reference extracts are used for TLC identity testing (compared with one of five CRS extracts in the European Pharmacopoeia). USP RS substances are predominantly used for assays (Table 1S, Supporting Information). Reagent qualities are never used for quantitation, but exclusively for identity testing, system suitability tests or peak identification. The requirements described for the quality of reagents are very meagre, on the other hand - in many cases, the reagents are not even listed in the United States Pharmacopoeia and, in other cases, the USP merely stipulates a "suitable grade". At the same time, the USP also uses three reference drugs, which are used for assays (Digitalis), identification by thin-layer chromatography (powdered ginger) or chemical identification (Rauwolfia serpentina). Six new monographs are currently planned and have already been published in the USP Pharmacopeial Forum on turmeric (Curcuma longa; turmeric; powdered turmeric; powdered turmeric extract; curcuminoids; curcuminoids capsules; curcuminoids tablets) and soy (Glycine max; powdered soy isoflavones extract; soy isoflavones tablets; soy isoflavones capsules) [33]. At the same time, four new reference standards have been established for turmeric, whereby the pure substances curcumin, bisdemethoxycurcumin und demethoxycurcumin are used for assays and the curcuminoid mixture is used for identity testing. As far as the planned soy monographs are concerned, a defatted soy reference extract is used for peak assignment and six new reference substances (daidzein, daidzin, genistein, genistin, glycitein and glycitin) are used for assays; a conversion factor is used to quantify the acetyl and malonyl derivatives.

The use of reference extracts for identity testing, as predominantly practiced in the USP, appears to be an expedient approach as it offers a means of obtaining a more informative fingerprint than can be obtained using pure substances alone. The use of reference extracts to identify peaks within a chromatographic process also appears to offer advantages, particularly in the EP monograph on Ginkgo dry extract refined and quantified, where the extract actually replaces no less than four pure substances, or in the planned USP monographs on soy, where the extract can be used to identify the acetyl and malonyl derivatives of the isoflavons, which are not available as pure substances. Also in the EP monograph on fresh bilberry fruit dry extract, refined and standardised the CRS extract allows the identification of 20 signals in the HPLC chromatogram which offers a big advantage over the use of individual reference substances. As far as the EP monographs on ivy leaf, milk thistle fruit and extract, valerian root, extract and tincture and chaste tree fruit are concerned, on the other hand, the use of reference extracts for quantitation of the markers does not appear expedient on the face of it, as the pertinent identity tests always resort to pure substances, which would all be available commercially at prices that are comparable with those of the CRS extracts. The only extract to offer any advantages is the milk thistle reference extract, as it enables identification of silychristin, silydianin and isosilybinin as well as quantitation (as silybinin). Nevertheless, quantitation could also be carried out satisfactorily against a silybinin reference substance as in the pertinent USP monograph, which also resorts to casticin, valerenic acid and silybinin in the respective monographs on chaste tree, valerian and milk thistle. A summarised appraisal of the current European Pharmacopoeia CRS extracts according to the criteria 
Table 2 CRS reference substances in the European Pharmacopoeia (Issue 6.2).

\begin{tabular}{|c|c|c|c|c|c|}
\hline CRS reference substance in EP & Monograph \# & Monograph & Identity & $\begin{array}{l}\text { Purityl } \\
\text { limit }\end{array}$ & Assay \\
\hline Arbutin & 1054 & Bearberry leaf & & & $x$ \\
\hline Benzyl alcohol & 1827 & Ginkgo dry extract refined and quantified & & & $x$ \\
\hline \multirow[t]{2}{*}{ Boldine } & 1396 & Boldo leaf & & & $x$ \\
\hline & 1816 & Boldo leaf dry extract & & & $x$ \\
\hline Caffeine & 1504 & Cola & & & $x$ \\
\hline \multirow[t]{3}{*}{ Capsaicin } & 1859 & Capsicum & & $x$ & $x$ \\
\hline & 2336 & Capsicum oleoresin refined and quantified & & $x$ & $x$ \\
\hline & 2337 & Capsicum tincture standardised & & $x$ & $x$ \\
\hline \multirow[t]{4}{*}{ Cephaeline hydrochloride } & 0094 & Ipecacuanh root & $\mathrm{x}$ & & \\
\hline & 1875 & Ipecacuanha liquid extract standardised & $\mathrm{x}$ & & \\
\hline & 0093 & Ipecacuanha prepared & $\mathrm{x}$ & & \\
\hline & 1530 & Ipecacuanha tincture standardised & $x$ & & \\
\hline \multirow[t]{6}{*}{ Chlorogenic acid } & 1866 & Artichoke leaf & $x$ & & $\mathrm{x}$ \\
\hline & 1821 & Narrow-leaved coneflower root & $\mathrm{x}$ & & $\mathrm{x}$ \\
\hline & 1897 & Nettle leaf & $x$ & & $x$ \\
\hline & 1822 & Pale coneflower root & $x$ & & $x$ \\
\hline & 1823 & Purple coneflower herb & $x$ & & $x$ \\
\hline & 1824 & Purple coneflower root & & & $x$ \\
\hline Coumarin & 2120 & Melilot & $\mathrm{x}$ & & $x$ \\
\hline Cyanidin chloride & 2394 & $\begin{array}{l}\text { Fresh bilberry fruit dry extract refined and stan- } \\
\text { dardised }\end{array}$ & & $x$ & \\
\hline Digitoxin & 0117 & Digitalis leaf & & & $x$ \\
\hline \multirow[t]{4}{*}{ Emetine hydrochloride } & 0094 & Ipecacuanha root & $x$ & & \\
\hline & 1875 & Ipecacuanha liquid extract standardised & $x$ & & \\
\hline & 0093 & Ipecacuanha prepared & $\mathrm{x}$ & & \\
\hline & 1530 & Ipecacuanha tincture standardised & $\mathrm{x}$ & & \\
\hline Ferulic acid & 1834 & Lemon verbena leaf & & & $x$ \\
\hline Foeniculin for peak identification & 0804 & Anise oil & & $x$ & \\
\hline Ginkgolic acids & 1827 & Ginkgo dry extract refined and quantified & & & $\mathrm{x}$ \\
\hline \multirow[t]{3}{*}{ Glycyrrhizate (monoammonium) } & 2378 & Liquorice dry extract for flavouring purposes & & & $\mathrm{x}$ \\
\hline & 1536 & Liquorice ethanolic liquid extract standardised & & & $\mathrm{x}$ \\
\hline & 0277 & Liquorice root & & & $x$ \\
\hline \multirow[t]{2}{*}{ Harpagoside } & 1871 & Devil's claw dry extract & & & $x$ \\
\hline & 1095 & Devil's claw root & & & $x$ \\
\hline Oleuropein & 1878 & Olive leaf & & & $\mathrm{x}$ \\
\hline Purpureaglycoside A & 0117 & Digitalis leaf & $\mathrm{x}$ & & \\
\hline Purpureaglycoside B & 0117 & Digitalis leaf & $\mathrm{x}$ & & \\
\hline Quercetin dihydrate & 1827 & Ginkgo dry extract refined and quantified & & & $x$ \\
\hline Ruscogenins & 1847 & Butcher's broom & $\mathrm{x}$ & & $x$ \\
\hline \multirow[t]{2}{*}{ Rutoside trihydrate } & 2184 & Buckwheat herb & & & $x$ \\
\hline & 1874 & St. John's wort dry extract quantified & & & \\
\hline \multirow[t]{2}{*}{ Salicin } & 1583 & Willow bark & & & $x$ \\
\hline & 2312 & Willow bark dry extract & & & $\mathrm{x}$ \\
\hline Verbenalin & 1854 & Verbena herb & & & $x$ \\
\hline
\end{tabular}

mentioned above is given in $\bigcirc$ Table $\mathbf{1}$, as well as a comparison with the USP.

\section{Future Developments and Challenges within the EP and USP}

The EP and the USP are constantly developing new monographs on herbal drugs and herbal drug preparations and it will be a big challenge to select suitable CRS reference substances, CRS extracts and authentic herbal drug materials to be applied in assays or system suitability tests.

The USP Dietary Supplements Expert Committee (DSB EC) has currently identified the following exemplary candidates for future monographs: mangosteen, noni, Aloe vera gel, cranberry extract, cinnamon, artichoke, elder blossom and elderberry, olive leaf, linseed, evening primrose oil, Stevia and passion flower. In addition to this, a number of monographs from Ayurvedic medicine, such as Andrographis, Ashwagandha, Boswellia, Garcinia and Guggul, are being adapted for the USP in cooperation with the Indian Pharmacopoeia. These monographs are currently in various stages of development; draft monographs on guggul, native guggul extract, purified guggul extract and guggul tablets were recently published in the Pharmacopoeial Forum [34]. Furthermore, monographs from Traditional Chinese Medicine (TCM) are being selected for inclusion in the USP in collaboration with the Chinese Pharmacopoeia.

The EP currently also makes considerable efforts in the field of Traditional Chinese Medicine. Approximately 100 TCM monographs are currently under development.

Apart from establishing reference extracts, the European Pharmacopoeia Commission is currently working towards replacing 
Table 3 Reagents used for assays in the European Pharmacopoeia (Issue 6.2).

\begin{tabular}{|c|c|c|c|c|c|}
\hline Reagent $R$ in EP & $\begin{array}{l}\text { Mono- } \\
\text { graph \# }\end{array}$ & Monograph & Method & $\begin{array}{l}\text { Required } \\
\text { purity }\end{array}$ & Remarks \\
\hline 11-Keto-beta-boswellic acid & 2310 & Indian frankincense & HPLC & $90 \%(\mathrm{HPLC})$ & percentage purity taken into consideration \\
\hline $\begin{array}{l}\text { Acetyl-11-keto-beta-bos- } \\
\text { wellic acid }\end{array}$ & 2310 & Indian frankincense & HPLC & $90 \%(\mathrm{HPLC})$ & percentage purity taken into consideration \\
\hline Apigenin 7-glucoside & 0404 & Matricaria flower & HPLC & $95 \%(\mathrm{HPLC})$ & percentage purity taken into consideration \\
\hline Asiaticoside & 1498 & Centella & HPLC & $97 \%$ (HPLC) & HPLC purity taken into consideration \\
\hline Berberine chloride & 1831 & Goldenseal rhizome & HPLC & $95 \%(\mathrm{HPLC})$ & $\begin{array}{l}\text { content calculation allows for percentage } \\
\text { of chloride-free berberine }\end{array}$ \\
\hline Capric acid & 1848 & Saw palmetto fruit & GC & $98 \%(G C)$ & percentage purity taken into consideration \\
\hline Caproic acid & 1848 & Saw palmetto fruit & GC & $98 \%(G C)$ & percentage purity taken into consideration \\
\hline \multirow[t]{3}{*}{ Cinchonine } & 0174 & Cinchona bark & $\begin{array}{l}\text { spectro- } \\
\text { photo- } \\
\text { metry }\end{array}$ & - & \\
\hline & & Cinchona liquid extract standardised & & & \\
\hline & 1818 & standardised & & & \\
\hline Eugenol & 2094 & $\begin{array}{l}\text { Oriental cashew for homoeopathic } \\
\text { preparations }\end{array}$ & $\begin{array}{l}\text { spectro- } \\
\text { photo- } \\
\text { metry }\end{array}$ & - & \\
\hline Ferulic acid & 1419 & Eleutherococcus & HPLC & $99 \%(\mathrm{HPLC})$ & $\begin{array}{l}\text { reagent used in spite of ferulic acid CRS } \\
\text { being available }\end{array}$ \\
\hline \multirow[t]{2}{*}{ Ginsenoside Rb1 } & 1523 & Ginseng & HPLC & $95 \%(\mathrm{HPLC})$ & HPLC purity taken into consideration \\
\hline & 2383 & Notoginseng root & & & \\
\hline \multirow[t]{2}{*}{ Ginsenoside Rg1 } & 1523 & Ginseng & HPLC & 95\% (HPLC) & HPLC purity taken into consideration \\
\hline & 2383 & Notoginseng root & & & \\
\hline Hederacoside C & 2092 & $\begin{array}{l}\text { Hedera helix for homoeopathic } \\
\text { preparations }\end{array}$ & HPLC & $95 \%(\mathrm{HPLC})$ & percentage purity taken into consideration \\
\hline Hydrastine hydrochloride & 1831 & Goldenseal rhizome & HPLC & $98 \%(\mathrm{HPLC})$ & $\begin{array}{l}\text { content calculation allows for percentage } \\
\text { of chloride-free hydrastine }\end{array}$ \\
\hline Lauric acid & 1848 & Saw palmetto fruit & GC & $98 \%(G C)$ & percentage purity taken into consideration \\
\hline Linoleic acid & 1848 & Saw palmetto fruit & GC & $98 \%(G C)$ & percentage purity taken into consideration \\
\hline Linolenic acid & 1848 & Saw palmetto fruit & GC & $98 \%(\mathrm{GC})$ & percentage purity taken into consideration \\
\hline Marrubiin & 1835 & White horehound & HPLC & $95 \%(G C)$ & percentage purity taken into consideration \\
\hline Oleic acid & 1848 & Saw palmetto fruit & GC & $98 \%(G C)$ & percentage purity taken into consideration \\
\hline Palmitic acid & 1848 & Saw palmetto fruit & GC & $98 \%(G C)$ & percentage purity taken into consideration \\
\hline Palmitoleic acid & 1848 & Saw palmetto fruit & GC & $98 \%(G C)$ & percentage purity taken into consideration \\
\hline Parthenolide & 1516 & Feverfew & HPLC & $90 \%$ (HPLC) & purity not taken into consideration \\
\hline Quercetin dihydrate & 1828 & Ginkgo leaf & HPLC & $90 \%(\mathrm{HPLC})$ & $\begin{array}{l}\text { content calculation allows for percentage } \\
\text { of anhydrous quercetin. Reagent used in } \\
\text { spite of quercetin dihydrate CRS being } \\
\text { available }\end{array}$ \\
\hline \multirow[t]{3}{*}{ Quinine } & 0174 & Cinchona bark & $\begin{array}{l}\text { spectro- } \\
\text { photo- } \\
\text { metry }\end{array}$ & - & \\
\hline & & Cinchona liquid extract standardised & & & \\
\hline & 1818 & & & & \\
\hline Sinensetin & 1229 & Java tea & HPLC & $95 \%$ (HPLC) & purity not taken into consideration \\
\hline Stearic acid & 1848 & Saw palmetto fruit & GC & $98 \%(G C)$ & percentage purity taken into consideration \\
\hline
\end{tabular}

older, rather unspecific analytical techniques (thin-layer chromatographic methods; spectrophotometric assays) with new specific methods, usually HPLC. This particularly affects drugs containing anthraquinones and flavonoids (e.g., senna, rhubarb, alder buckthorn, as well as birch, passion flower and hawthorn). The establishment of new methods will also require the establishment of new CRS standards in some cases. This may also lead to an adjustment of the content values in certain monographs, as the determined content not only depends on the reference standard used, but may also be influenced to a very great extent by the method adopted. One example of this is aescine, a mixture of more than 30 different triterpene saponins. The aescine content in dry horse-chestnut extract has been determined by means of spectrophotometric analysis after derivatization, or ti- trimetric analysis up to now. This photometric method will be replaced by an HPLC technique in the new EP monograph, which is only capable of detecting a certain fraction of the saponins in the extract, so that the aescine content in the drug will be lower than the content determined by means of the spectrophotometric or titrimetric method. Plans now include a three-year transition period, in which the results of the new HPLC method are to be collected alongside the old, but still obligatory, photometric technique. When the resulting data has been evaluated, an adjustment of the content specifications is planned with a transition to the specific HPLC method [35].

A similar course of action may be expected for the anthraquinone drugs as a changeover from a photometric technique to an HPLC method could result in a significant alteration in the content val- 
Table 4 CRS reference extracts in the US Pharmacopoeia (up to and including USP31-NF 26 Second Supplement).

\begin{tabular}{|c|c|c|c|}
\hline USP reference extract & Monograph & Identity & Content \\
\hline \multirow[t]{5}{*}{ Powdered American Ginseng Extract } & Dietary Supplements American Ginseng & $\mathrm{x}$ & $\mathrm{x}$ \\
\hline & Dietary Supplements American Ginseng Capsules & $\mathrm{x}$ & $\mathrm{x}$ \\
\hline & Dietary Supplements American Ginseng Tablets & $x$ & $\mathrm{x}$ \\
\hline & Dietary Supplements Powdered American Ginseng & $\mathrm{x}$ & $\mathrm{x}$ \\
\hline & Dietary Supplements Powdered American Ginseng Extract & $\mathrm{x}$ & $\mathrm{x}$ \\
\hline \multirow[t]{9}{*}{ Powdered Asian Ginseng Extract } & Dietary Supplements American Ginseng & $x$ & $\mathrm{x}$ \\
\hline & Dietary Supplements American Ginseng Capsules & $\mathrm{x}$ & $\mathrm{x}$ \\
\hline & Dietary Supplements American Ginseng Tablets & $x$ & $\mathrm{x}$ \\
\hline & Dietary Supplements Asian Ginseng & $\mathrm{x}$ & $\mathrm{x}$ \\
\hline & Dietary Supplements Asian Ginseng Tablets & $\mathrm{x}$ & $\mathrm{x}$ \\
\hline & Dietary Supplements Powdered American Ginseng & $\mathrm{x}$ & $\mathrm{x}$ \\
\hline & Dietary Supplements Powdered American Ginseng Extract & $x$ & $\mathrm{x}$ \\
\hline & Dietary Supplements Powdered Asian Ginseng & $\mathrm{x}$ & $\mathrm{x}$ \\
\hline & Dietary Supplements Powdered Asian Ginseng Extract & $\mathrm{x}$ & $\mathrm{x}$ \\
\hline Powdered Bilberry Extract & Dietary Supplements Powdered Bilberry Extract & $\mathrm{x}$ & $x($ Peak ID) \\
\hline \multirow[t]{5}{*}{ Powdered Black Cohosh Extract } & Dietary Supplements Black Cohosh & $\mathrm{x}$ & $x($ Peak ID) \\
\hline & Dietary Supplements Black Cohosh Fluid Extract & $x$ & $x($ Peak ID) \\
\hline & Dietary Supplements Black Cohosh Tablets & $\mathrm{x}$ & $x($ Peak ID $)$ \\
\hline & Dietary Supplements Powdered Black Cohosh & $\mathrm{x}$ & $x($ Peak ID) \\
\hline & Dietary Supplements Powdered Black Cohosh Extract & $\mathrm{x}$ & $x($ Peak ID) \\
\hline \multirow[t]{5}{*}{ Powdered Cat's Claw Extract } & Dietary Supplements Cat's Claw & $x$ & $x($ Peak ID) \\
\hline & Dietary Supplements Cat's Claw Capsules & $\mathrm{x}$ & $x($ Peak ID) \\
\hline & Dietary Supplements Cat's Claw Tablets & $x$ & x (Peak ID) \\
\hline & Dietary Supplements Powdered Cat's Claw & $\mathrm{x}$ & $x($ Peak ID) \\
\hline & Dietary Supplements Powdered Cat's Claw Extract & $\mathrm{x}$ & $x($ Peak ID) \\
\hline Powdered Decaffeinated Green Tea Extract & Dietary Supplements Powdered decaffeinated green tea extract & $x$ & x (Peak ID) \\
\hline \multirow[t]{3}{*}{ Powdered Echinacea angustifolia Extract } & Dietary Supplements Echinacea angustifolia & $\mathrm{x}$ & \\
\hline & Dietary Supplements Powdered Echinacea angustifolia & $\mathrm{x}$ & \\
\hline & Dietary Supplements Powdered Echinacea angustifolia extract & $\mathrm{x}$ & \\
\hline \multirow[t]{3}{*}{ Powdered Echinacea pallida Extract } & Dietary Supplements Echinacea pallida & $\mathrm{x}$ & \\
\hline & Dietary Supplements Powdered Echinacea pallida & $\mathrm{x}$ & \\
\hline & Dietary Supplements Powdered Echinacea pallida Extract & $\mathrm{x}$ & \\
\hline \multirow[t]{4}{*}{ Powdered Echinacea purpurea Extract } & Dietary Supplements Echinacea purpurea Aerial parts & $\mathrm{x}$ & \\
\hline & Dietary Supplements Echinacea purpurea Root & $\mathrm{x}$ & \\
\hline & Dietary Supplements Powdered Echinacea purpurea & $\mathrm{x}$ & \\
\hline & Dietary Supplements Powdered Echinacea purpurea Extract & $\mathrm{x}$ & \\
\hline \multirow[t]{3}{*}{ Powdered Eleuthero Extract } & Dietary Supplements Eleuthero & & $\mathrm{x}$ \\
\hline & Dietary Supplements Powdered Eleuthero & & $\mathrm{x}$ \\
\hline & Dietary Supplements Powdered Eleuthero Extract & & $\mathrm{x}$ \\
\hline \multirow[t]{5}{*}{ Powdered Milk Thistle Extract } & Dietary Supplements Milk Thistle & $\mathrm{x}$ & $x($ Peak ID) \\
\hline & Dietary Supplements Milk Thistle Capsules & $x$ & $x($ Peak ID) \\
\hline & Dietary Supplements Milk Thistle Tablets & $x$ & $x($ Peak ID) \\
\hline & Dietary Supplements Powdered Milk Thistle & $\mathrm{x}$ & x (Peak ID) \\
\hline & Dietary Supplements Powdered Milk Thistle Extract & $\mathrm{x}$ & $x($ Peak ID) \\
\hline \multirow[t]{4}{*}{ Powdered Red Clover Extract } & Dietary Supplements Powdered Red Clover & $\mathrm{x}$ & $x($ Peak ID) \\
\hline & Dietary Supplements Powdered Red Clover Extract & $\mathrm{x}$ & $x($ Peak ID) \\
\hline & Dietary Supplements Red Clover & $x$ & $x($ Peak ID) \\
\hline & Dietary Supplements Red Clover Tablets & $x$ & $x($ Peak ID) \\
\hline \multirow[t]{3}{*}{ Powdered St. John's Wort Extract } & Dietary Supplements Powdered St. John's Wort & & $x($ Peak ID) \\
\hline & Dietary Supplements Powdered St. John's Wort Extract & $x$ & x (Peak ID) \\
\hline & Dietary Supplements St. John's Wort & & $x($ Peak ID) \\
\hline \multirow[t]{3}{*}{ Powered Chaste Tree Extract } & Dietary Supplements Chaste Tree & $\mathrm{x}$ & \\
\hline & Dietary Supplements Powdered Chaste Tree & $\mathrm{x}$ & \\
\hline & Dietary Supplements Powdered Chaste Tree Extract & $\mathrm{x}$ & \\
\hline \multirow[t]{3}{*}{ Pygeum Extract } & Dietary Supplements Pygeum & $\mathrm{x}$ & \\
\hline & Dietary Supplements Pygeum Capsules & $\mathrm{x}$ & \\
\hline & Dietary Supplements Pygeum Extract & $\mathrm{x}$ & \\
\hline Tomato Extract Containing Lycopene & Dietary Supplements Tomato Extract Containing Lycopene & & $\mathrm{x}$ \\
\hline
\end{tabular}


ues. One example of this is the USP sennoside standard for photometric determination, which exhibits a purity of just around 75\% according to HPLC analysis.

A further example for the strong dependence between the assigned content of a reference standard and the applied analytical methodology is glycyrrhizic acid as a CRS reference substance. In the EP liquorice monographs an HPLC method with UV detection is applied, while in the planned monograph on Cimicifuga an HPLC method with ELSD detection is described. Although the same CRS is used - glycyrrhizate (monoammonium) CRS - different content values will need to be assigned to the CRS. These result from the different detection systems and thus from the overall low purity of the CRS itself, which currently has an assigned content of $78.6 \%$ only. The application of a more pure glycyrrhizic acid should reduce the observed differences as influence of the contained impurities would be minimised.

\section{Reference Substances in Pharmacological] Toxicological Research \\ $\nabla$}

Another crucial point in respect to the topic of reference compounds is their use for pharmacological and toxicological investigations. Regardless of whether commercially available or inhouse isolated compounds are assayed, it is current practice to define their purity by conventional (e.g., chomatographic, titrimetric or photometric) methods, mostly leaving two major questions unanswered. Firstly, is the selected analytical method the most suitable one to determine not only the major compound but also possible impurities, and secondly, to what extent do impurities contribute to the overall pharmacological effect (or are impurities responsible for activity actually)? Discussing this highly relevant problem in detail is not really within the scope of this review. But the few above-mentioned statements should increase the reader's awareness in this respect, especially as they might explain the commonly observed problem of nonreproducible pharmacological test results in part.

This issue was also recently discussed in the AOAC reference standard newsletter [36].

\section{Conclusions}

$\nabla$

Well characterised reference standards constitute the basis for the informative and reliable quality inspection and testing as well as for the pharmacological and toxicological characterisation of herbal products. The terms "reference" and "standard" alone imply a measure of reliability that is at least appropriate for the scale of the problem concerned. This scale may take on considerable proportions in both the pharmaceutical and the food sector, where the testing of parameters affecting safety is concerned.

The provision of appropriately qualified reference standards of herbal constituents will continue to present a challenge in the future because of numerous special aspects relating to their sources and applications. At the same time, there are extensive national and international rules and regulations which must be observed within the framework of quality inspection and testing of herbal products. These should be standardised to a greater extent in view of the increasing international trade of herbs and herbal products. The harmonisation of specifications in authoritative pharmacopoeias (e.g., EP, USP, JP) could constitute a step in this direction in analogy to the recently achieved harmonisation of microbiological testing procedures. The establishment of consolidated reference standards would then be a logical step. The establishment of reference extracts instead of chemically defined CRS for quantitative assays in the EP is not undisputed. The applicability and informational value of this approach should be investigated very carefully during the preliminary stages in each individual case. On the other hand it would appear appropriate to limit the still very common use of pharmacopoeial "reagents" as reference substances for quantitative determination in the future and replace them with appropriately characterised CRS standards.

Technical advances in the fields of NMR spectroscopy and mass spectrometry over the last years now enable the identification of marker substances in very low concentrations and therefore at a very early stage in the development of herbal preparations. Quantitative NMR spectroscopy is a valuable option for purity assessment and content assignment of reference standards. However, while providing a similar level of accuracy and precision as compared to "classical" analytical techniques, e.g., HPLC, it does not eliminate the necessity of relying on relative benchmarks to determine the purity and content of reference substances.

The provision of standards that are suitable for the characterisation of herbal preparations with complex mixtures of active substances, such as anthranoid drugs or horse-chestnut extract, will constitute a particular challenge in the years to come, if the content information that has been previously based on conventional methods is to be determined by means of selective methods in the future.

In the field of pharmacological and toxicological research the awareness should be increased for the importance of the quality of reference standards. While blatantly distorted results due to inadequately qualified reference standards and test substances may be an exception rather than a rule, the issue should be considered in view of the potentially far reaching consequences of pharmacological/toxicological test results. Appropriate recommendations by the authoritative scientific associations could constitute a step in this direction.

\section{Acknowledgements}

$\nabla$

The authors wish to thank Dr. Joseph Zapp (Universität des Saarlandes, Saarbrücken, Germany) for his contribution to the paragraph on qNMR spectroscopy, Josef Brinckmann of Traditional Medicinals, Sebastopol, CA, USA, and Dr. Maged Sharaf, USP (USA) for information concerning USP reference substances and activities, and Dr. Klaus Reif of PhytoLab GmbH \& Co. KG (Vestenbergsgreuth, Germany) and Dr. Sven Oliver Kruse of Diapharm Analytics GmbH (Oldenburg, Germany) for for their valuable comments on this article.

\section{References}

1 Reif K, Sievers H, Steffen J-P. The role of chemical reference standards as analytical tools in the quality assessment of botanical materials - a European perspective. Herbal Gram 2004; 63: 38-43

2 Helliwell K. Herbal reference standards. Pharmeuropa 2006; 2: 235238

3 Veit $M$, Wissel S. Definitionen und Anforderungen an Referenzstandards in der Arzneimittelprüfung. Pharm Ind 2007; 12: 1475-1480

4 Veit $M$, Wissel S. Referenzstandards in der Arzneimittellprüfung. Pharm Ind 2008; $1: 135-138$

5 Dietary supplements and health education act, Public Law 103-417, 103rd Congress, 1994. Available at http://www.fda.gov/opacom/laws/ DSHEA.html. Accessed November 27, 2008 
6 Current good manufacturing practice in manufacturing, packaging, labeling, or holding operations for dietary supplements; Final Rule, 21 CFR Part 111, 2007. Available at http://www.cfsan.fda.gov/\%7Elrd/ fr07625a.html. Accessed November 27, 2008

7 Safety assessment of botanicals and botanical preparations intended for use as ingredients in food supplements, guidance document of the Scientific Committee (Question No EFSA-Q-2005-233), European Food Safety Authority, 2007. Available at http://www.efsa.europa.eu/EFSA/ efsa_locale-1178620753812_1178717026833.htm. Accessed November 27, 2008

8 European Commission request to the European Food Safety Authority for scientific advice on: the community list of permitted health claims pursuant article 13 of regulation 1924/2006 on nutrition and health claims made on foods - terms of reference, EFSA, 2008. Available at http://www.efsa.europa.eu/cs/BlobServer/General/claims_art13_ TOR,3.pdf?ssbinary=true. Accessed November 27, 2008

9 European Pharmacopeia Edition 6.2. Available at http://www.edqm. org. Accessed November 27, 2008

10 United States Pharmacopeia - National Formulary. Available at www. uspnf.com. Accessed November 27, 2008

11 Note for guidance on good manufacturing practice for active pharmaceutical ingredients (CPMP/ICH/4106/00), ICH Topic Q7, good manufacturing practice for active pharmaceutical ingredients. Available at http://www.emea.europa.eu/pdfs/human/ich/410600en.pdf. Accessed November 27, 2008

12 ISO Guide 30. Terms and definitions used in connection with reference materials. Geneva: International Organisation for Standardization; 1992

13 ISO Guide 31. Reference materials. Contents of certificates and labels. Geneva: International Organisation for Standardization; 2000

14 ISO Guide 32. Calibration in analytical chemistry and use of certified reference materials. Geneva: International Organisation for Standardization; 1997

15 ISO Guide 33. Uses of certified reference materials. Geneva: International Organisation for Standardization; 2000

16 ISO Guide 34. General requirements for the competence of reference materials producers. Geneva: International Organisation for Standardization; 2000

17 ISO Guide 35. Reference materials. General and statistical principles for certification. 2006. Geneva: International Organization for Standardization; 2006

18 Guideline on quality of herbal medicinal products/traditional herbal medicinal products (CPMP/QWP/2819/00 Rev 1). Available at http:// www.emea.europa.eu/pdfs/human/qwp/281900en.pdf. Accessed November 27, 2008

19 Guideline on specifications: test procedures and acceptance criteria for herbal substances, herbal preparations and herbal medicinal products/ traditional herbal medicinal products (CPMP/QWP/2820/00 Rev 1). Available at http://www.emea.europa.eu/pdfs/human/qwp/282000en. pdf. Accessed November 27, 2008

20 Guidance for industry. Botanical drug products, U.S. Department of Health and Human Services, Food and Drug Administration, June 2004

21 Reflection paper on markers used for quantitative and qualitative analysis of herbal medicinal products and traditional herbal medicinal products (EMEA/HMPC/253629/2007). Available at http://www.emea. europa.eu/pdfs/human/hmpc/25362907enfin.pdf. Accessed January 30,2009

22 NIST/EPA/NIH (NIST 08) Mass Spectral Library. National Institute of Technology. Available at http://www.nist.gov/srd/nist1a.htm. Accessed November 27, 2008

23 Spectral Database for Organic Compounds. National Institute of Advanced Industrial Science and Technology (AIST). Available at http://riodb01.ibase.aist.go.jp/sdbs/cgi-bin/cre_index.cgi?lang=eng. Accessed November 27, 2008

24 ICH Topic Q 2 (R1) Validation of analytical procedures: text and methodology (CPMP/ICH/381/95). 1995. Available from http://www.emea. europa.eu/pdfs/human/ich/038195en.pdf. Accessed November 27, 2008

25 Pauli G, Jaki U, Lankin D, Walter J, Burton I. Quantitative NMR of bioactive natural products. In: Colegate $\mathrm{S}$, Molyneux R, editors. Bioactive natural products. Boca Raton: CRC Press; 2008: 113-141

26 Diehl BWK, Malz F, Holzgrabe U. Quantitative NMR spectroscopy in the quality evaluation of active pharmaceutical ingredients and excipients. Spectrosc Eur 2007; 19: 15-19

27 Pauli G, Jaki B, Lankin D. Quantitative H NMR: development and potential of a method for natural products analysis. J Nat Prod 2005; 68: 133-149

28 Lopez-Rituerto E, Cabredo S, Lopez M, Avenoza A, Busto JH, Peregrina JM. A thorough study on the use of quantitative ${ }^{1} \mathrm{H}$ NMR in rioja red wine fermentation process. J Agric Food Chem 2009; 57: 2112-2118

29 Bekiroglu S, Myrberg O, Oestman K, Ek M, Arvidsson T, Rundloef T, Hakkarainen $B$. Validation of a quantitative NMR method for suspected counterfeit products exemplified on determination of benzethonium chloride in grapefruit seed extracts. J Pharm Biomed Anal 2008; 47: 958-961

30 Mohn T, Cutting B, Ernst B, Hamburger M. Extraction and analysis of intact glucosinolates - a validated pressurized liquid extraction/liquid chromatography - mass spectrometry protocol for Isatis tinctoria, and qualitative analysis of other cruciferous plants. J Chromatogr A 2007; 1166: $142-151$

31 Mehr K, John B, Russel D, Avizonis D. Electronic referencing techniques for quantitative NMR: pitfalls and how to avoid them using amplitudecorrected referencing through signal injection. Anal Chem 2008; 80: $8320-8323$

32 Quantifying uncertainty in analytical measurement. Eurachem/CITAC Guide CG 4, second edition. Available at http://www.measurementuncertainty.org/mu/QUAM2000-1.pdf. Accessed November 27, 2008

33 Turmeric; Powdered Turmeric; Powdered Turmeric Extract; Curcuminoids; Curcuminoids Capsules; Curcuminoids Tablets; Powdered Soy Isoflavones Extract; Soy Isoflavones Tablets; Soy Isoflavones Capsules. USP Pharmacopoeial Forum 2007: 6

34 Guggul, Native Guggul Extract, Purified Guggul Extract and Guggul Tablets. USP Pharmacopoeial Forum 2008: 4

35 Horse-chestnut/horse-chestnut dry extract, standardized. Pharmeuropa 2008 ; $20: 478-483$

36 Betz JM. The importance of reference materials to the dietary supplement research and manufacturing communities. AOAC, TDRM. The Reference Standard Newsletter 2008; 42: 1-3 